\title{
Téoros
}

Revue de recherche en tourisme

\section{Mutations culturelles et pratiques touristiques}

\section{André Hut}

Volume 4, numéro 2, juillet 1985

Les clientèles touristiques : un tour d'horizon

URI : https://id.erudit.org/iderudit/1080631ar

DOI : https://doi.org/10.7202/1080631ar

Aller au sommaire du numéro

Éditeur(s)

Université du Québec à Montréal

ISSN

0712-8657 (imprimé)

1923-2705 (numérique)

Découvrir la revue

Citer cet article

Hut, A. (1985). Mutations culturelles et pratiques touristiques. Téoros, 4(2),

31-33. https://doi.org/10.7202/1080631ar d'utilisation que vous pouvez consulter en ligne.

https://apropos.erudit.org/fr/usagers/politique-dutilisation/ 


\title{
Mutations culturelles et pratiques touristiques
}

\author{
par André Hut*
}

La naissance de nouveaux courants d'idées engendrent des nouvelles sensibilités et centres d'intérêts; parallèlement, apparaissent des initiatives socioculturelles et ludiques qui ont des répercussions sur les vacances et le tourisme soit que les visiteurs souhaitent les retrouver sur le terrain des vacances et des loisirs, soit que les populations d'ac cueii les aient elles-mêmes mises sur pied.

Cette évolution va influencer d'une manière originale et spécifique les conditionnements permanents qui caracterisent habituellement les choix et les comportements du vacancier dans l'utilisation de son temps ibre : I'origine géographique et urbaine, les conditions de travail et d'habitat, la catégorie d'áge, l'engagement personnel, la classe sociale, l'expérience acquise des vacances, les moyens financiers, les habitudes et choix préferentiels de consommation...

Les premiers et les seconds constitueraient les dimensions horizontales et verticales d'un tableau à double entrée.

\section{Crise de civilisation et ré-évaluations}

La crise qui caractérise ces dix dernières années ne se limite pas uniquement au secteur énergétique ni au domaine économique seulement: il s'agit aussi d'une crise de civilisation qui remet profondement en cause les valeurs sur lesquelles s'étaient bâties la société postindustrielle.

Brièvement et schématiquement, la mise en question des finalités et le renversement de valeurs évoquées pourraient se résumer comme suit :

- revendication de la dignité humaine, des droits de l'homme et des peuples affectant respect et promotion;

\footnotetext{
'André Hut est Belge. II a 25 ans d'expérience en animation touristique. Expert sciantifique en tourisme. Andrí Hut a agi fréquemment comme consultant et a couvré comme ensaignant et journaliste.
}

- redécouverte de soi-même dans sa globalité au-delá du seul rationnel (le corps, les moyens d'expression, les racines culturelles, la dimension de l'imaginaire, l'inconscient...):

- rencontre des autres dans la reconnaissance des différences et l'enrichissement de l'échange;

- remise en question du primat du travail et de l'économique incluant une ré-évaluation du temps libre et des autres dimensions de la vie sociale;

- mise en doute et même rejet des idéologies ainsi que des dogmatismes et des religions traditionnelles exacerbant la recherche intellectuelle et spirituelle, tout azimuth, de la signification profonde et du sens dernier des choses et de la vie.

A l'opposé, cependant, se développent des courants rétrogrades en faveur de l'apartheid, de la xénophobie et du racisme, des nationalismes exacerbés, des replis protectionnistes encouragés par la crise économique... comme aussi la renaissance de nouveaux dogmatismes et le regain d'options "fondamentalistes" des grandes religions du Livre: ... facteurs défavorables à un tourisme de rencontre, de dialogue et d'échanges.

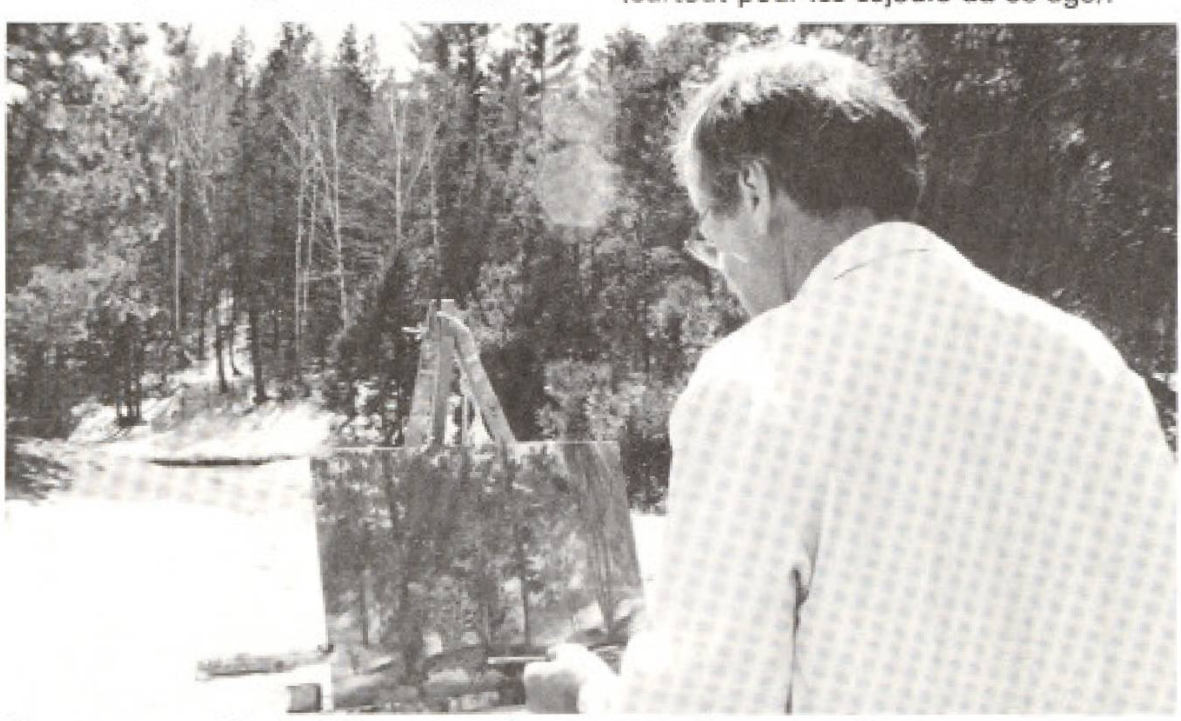

Un renouveau des intérêts culturels : stages, séjours spécialisés, apprentissage sur place chez l'artisan
II n'empêche que cette mutation positive restitue qualitativement au tourisme sa finalité socio-culturelle qu'un traitement unidimensionnellement économique aurait réduit à un produit, une marchandise à vendre : territoire, patrimoine et personnes en visite ou à l'accueil.

\section{Nouvelles pratiques ludiques, socio-culturelles et touristiques}

Ces diverses pratiques reflètent des aspirations nouvelles, un changement de mentalités à partir d'une sensibilité à d'autres valeurs. En fait, elles representent diverses facettes d'une revendication globale: la qualité de la vie.

\section{Recherche d'un équilibre psycho-somatique}

Dans ce but et pour lutter contre la sédentarisation et le stress des conditions de travail ainsi que de la vie urbaine : jogging individuel et cross ou marathons populaires; parcours de Sante; clubs de gymnastique, de yoga, d'aérobic se multiplient mais aussi sessions et stages de week-end ou de vacances pour initier ou perfectionner ces pratiques qui sont mêmes offertes comme une des activités possibles dans des centres de vacances du tourisme associatif ou commercial (surtout pour les séjours du $3 e$ age). 
Expressions et communications culturelles

Un renouveau des intérèts culturels se manifeste pour l'apprentissage et la pratique du chant collectif, d'un instrument de musique, de l'expression corporelle et de la danse, du Super 8 et de la vidéo, des jeux informatiques, de l'artisanat d'art... d’où stages, séjours spécialisés, festivals, apprentissage sur place chez l'artisant, etc.

\section{Ecologie, environnement et tourisme vert}

- Le mouvement écologique a fait prendre conscience de l'importance de l'environnement physique et social à conserver, protéger et promouvoir, en tenant compte du caractère non-renouvelable de certaines réalitês, de l'équilibre des éco= systèmes à respecter, du gaspillage scandaleux provoqué par la société de consommation, du danger de catastrophe nucléairel Cette prise de conscience veut s'accompagner de l'implication des citoyens et des usagers eux-memes, invités à changer leur comportement individuel et à agir collectivement sur la scène publique.

- D'où attention privilégiée envers les sites et lieux naturels (littoraux, riviêres, marais, grottes, bois, baies, microclimats... ou humainement influencés Imaisons, fermes, champs, prairies, forêts, etangs, parcs et jardins...).

- En conséquence, des associations volontaires actives dans ce domaine font naître des maisons et musées de la forêt, des centres d'information ou d'interprétation de la nature (USA-Canada), des réserves fauniques et parcs à gibiers indigènes, des parcs naturels et centres d'initiation aux réalités naturelles et humaines présentes... Les administrations des Eaux et Forêts (Europe) ou des ministères de l'Énergie (Québec) prennent des initiatives en faveur d'un acces ludique et éducatif de la forêt - a dimension préventive - grâce à la construction de parkings, pavillons de halte et de relais accompagnés de centres d'information avec exposition, documentation, outils audiovisuels: en outre, promenades guidées et safaris-photos dans les campagnes, la forat, les fagnes, la montagne... avec un souci educatif pluri-disciplinaire; d'où formation de guides nature, ad hoc...

- Dans le même sens apparaissent une philosophie, des activités et bases de séjour axées sur le plein air, dans la conception québécoise du terme, qui dépasse la dimension restreinte a la seule activité sportive trop orientée par la compétition et le professionnalisme.

- Enfin, le développement du tourisme rural ou tourisme vert se manifeste partout.
Promenades et circuits de découvertes

- Certaines associations se spécialisent dans l'organisation, le week-end et durant les congés, de promenades à pied, à vélo, en ski, a cheval, et en voiture dans le sens d'une découverte des régions et de la population qui y habite.

- D'où randonnées pédestres et équestres, cyclo-tourisme, parcours de ski de fond, routes à theme pour voiture familiale (polyvalentes ou spécialisées), remise sur pied d'anciennes lignes de tramways oú de chemins de fer devenant trains touristiques, location de roulottes hyppomobiles, de péniches équipées naviguant sur les canaux, les fleuves ou les étendues marécageuses, circuits de canöes-kayaks avec équipements de camping et haltes-relais, sentiers de grandes randonnées et trechs de longue durée en montagne...

- Ces activités se démocratisent de plus en plus et sont accessibles à un premier niveau à des personnes peu initiées et à faibles movens; certaines demandent dejả un apprentissage et coûtent plus cher; dans ce dernier sens, il faut mentionner les clubs, sejours et formations en plongée sous-marine, spéléologie, alpinisme, parachutisme, voile, deltaplane...

\section{Histoire et racines socio-culturelles}

- Engouement pour l'histoire, I'archéologie, la recherche généalogique... que prouvent le succès des revues spécialisées, les records de vente de livres historiques et d'audition d'émissions de télévision spécialisées en ces matières.

- Des cercles locaux publient des études et présentent des expositions sur le passé et les activités socio-économiques de la population du village ou de la commune: une nouvelle muséologie se développe et se définit comme populaire parce qu'elle est soucieuse de mettre en valeur les personnes et les populations comme sujets et acteurs de l'histoire plutôt que les objets; d'où musées du marbre, du verre, du fer, de la pierre, de la laine, de la mine (charbon) mais aussi de la vie rurale ou de lexploitation forestière, de la pêche... qui révèlent les conditions de travail et de vie ainsi que les luttes sociales des travailleurs de ces diverses activités socio-économiques; ainsi se multiplient aussi les aménagements d'archéologie industrielle ou rurales, dans les lieux mêmes de productions.

- En France, au Québec et en Belgique maissent des ecomusées ruraux, industriels ou urbains; en outre, dans le sens des traditions et arts populaires s'ouvrent des musées et expositions sur des éléments de la vie quotidienne qui, progressivement, dépassent le côté anecdotique et encyclopédique pour resituer les objets dans le contexte historique et social des diverses catégories de population: I'habitat, le costume, le jouet, l'affiche, l'enfant, les movens de transport...

- Sur un plan universitaire et scientifique. paraissent des recherches méthodologiques sur les mémoines collectives et les histoires de vie.

- Au niveau éducatif, la multiplication de movens pédagogiques : édition de guides commentés et illustrés, organisation de visites guidées sur le terrain là pied, vélo, rally-automobile ou autocars), confection de montages audio-visuels, cassettesvidéo ou valises pédagogiques, au service des enseignants et des groupes d'éducation populaire; afin de faciliter la diffusion et l'accessibilité, regroupement de ces outils, issus de graupes les plus divers, dans des médiathèques ou mème des centres de documentation populaires (cf. Québecl.

\section{Vulgarisation scientifique et tourisme technique}

- Comme pour l'histoire et souvent en recoupement avec le mouvement écologique, voici à nouveau un nouveau domaine en pleine croissance!

- En effet, la vulgarisation scientifique (T.V., revues spécialisées, expositions, etc.). l'acquisition scolaire et professionnelle de formation technique, l'éveil et la formation des consommateurs... aiguisent l'intérêt des gens, durant leurs loisirs, vacances comprises, pour la visite et la découverte guidées d'activités économiques (usines, artisanat, cooperatives, fermes et centres d'élevages, moulins, scieries et barrages, énergies alternatives...) ou scientifiques (centres de recherches en télé-communications, agronomie, astronomie, etc.): ainsi se développent diverses formes de tourisme technique et des formules portes-ouvertes à certains moments de l'année.

- Malheureusement, trop souvent, la présentation et les commentaires demeurent strictement et unidimensionnellement économiques ou scientifiques et n'abordent pas, fréquemment, d'une manière pluridisciplinaire, les aspects sociaux, écologiques, culturels ou politiques, sauf si les organisateurs utilisent ce moyen - non pas comme campagne de relations publiques à dimension commerciale et publicitaire - mais pour une sensibilisation des visiteurs ầ une réalité et â des revendications socio-politiques (éleveurs, vignerons, usines occupées, etc.) oủ â de nouvelles pratiques sociales a/ternatives de production ou de services, inaugurant de nouveaux rapports sociaux. de production et d'organisation. (ex. : initiatives de chômeurs face à la crise, mouvement communautaire, etc.) cf. Le tourisme socio-politique qui s'est dévelop- 
pé après la révolution des celllets au Portugal.

\section{Recherche spirituelle}

\section{et aide psycho-sociale}

- La crise des ideologies et la mise en question des dogmatismes, jointes a l'isolement et à lanonymat de la vie sociale actuelle - surtout urbaine - expliquent la nécessité et le succès des diverses initiatives d'ecoute anonyme, par téléphone ou porte-ouverte.

En outre, l'arrêt du temps de loisirs, la découverte de témoignages sur la précarité de la vie, des choses et des civilisations, la distanciation géographique et sociale de son milieu de vie habituelle... occasionnent au coeur même des vacances un réveil ou une accentuation des questionnements!

- Cela explique la fréquentation plus importantes des cellébrations liturgiques durant les vacances, les visites et consultations dans les abbayes, la création sur certaines côtes de France de redais spirituels ou haltes de prieres, lieux confessionnels ou pluralistes de réflexions et d'échanges, dans un style détendu, très ouvert, au climat sympathique et tolérant.

- En ce sens, les initiatives qui ont été prises par les Églises, dans le cadre de la pastorale du tourisme notamment dans la ligne du Congrès sur les valeurs spirituefles du tourisme, mis sur pied par l'UIOOT laujourd'hui l'OMTl ă Rome, en 1967 conjointement avec la Commission pontificale des migrations et du tourisme de l'Église catholique. cf. Le document de rOMT, récent, sur les valeurs spirituelles du tourisme.

\section{En conclusion}

II apparailt :

Une grande diversification d'initiatives et de propositions accompagnées d'une diversification dans la localisation beaucoup plus diffuse et éclatée.

En outre, l'allongement du temps libre lcongess payes, pré-retraites, scolarité prolongée, chômage forcé, etc.) joint à une revalorisation des loisirs, amene les usagers à répartir autrement leurs temps libres au cours de la journée, semaine, année ou vie et selon de nouveaux criteres de valeurs en faveur d'une ré-appropriation du temps (le temps choish), à reconsidérer sur un plan personnel ou collectif et comme revendication politique.

A cette diversification dans l'utilisation du temps libre s'ajoute le profil de plus en plus diversifié des personnes qui pratiquent les loisirs et le tourisme : a celles qui exercent pour la premiere fois des vacances, il faut opposer d'autres qui héritent d'une expérience de 4 générations deja; en outre, certains usagers s'adonnent surtout à l'une des activités relatées ci-dessus, d'autres en cumulent plusieurs ou mẻme toutes celles-ci.

A l'opposé, il faut observer la présence de facteurs centripèdes et unificateurs comme la qualité de la vie, la dimension socioéducative et culturelle de l'éducation permanente, la volonté d'une mise en place d'une politique générale des loisirs qui allierait trois composantes : la satisfaction de la demande et de l'offre de la population permanente, des loisirs de proximité pour les urbains ou ruraux proches, enfin les vacances pour les populations plus éloignées avec une utilisation diversifiée des mêmes équipements! $f$

\section{L'innovation touristique}

\section{par Jean-Marie Lucas-Girardville*}

L'innovateur est celui qui parvient à prouver ses ldees on les hiscrivant dans la réalite. Mais le monde ne demande pas à changer, il ne demande qúà se perpétuer. Diou le paradoxe de rinnovateur : il apporte un progrés que personne ne demande ${ }^{(1)}$.

Mais qu'est-ce tout d'abord que l'innovation? C'est en quelque sorte la création d'un objet nouveau ou d'une idée qui va transformer les habitudes de vie. Encore faut-il que cette idée ou cet objet puisse être concrétisé et qu'il réponde à un besoin véritable mais lațent chez nos marchés potentiels.

La difficulté réside justement dans le fait que ce besoin r'a généralement jamais été exprimé de façon formelle, et que l'idée innovatrice doit être prise en charge par un entrepreneur, qui a le goüt du risque, et un vendeur, les deux généralement differents de Finnovateur. Car celui-ci n'est généralement qu'un créateur et un expérimentateur.

Ce qui rend I'innovation si difficile, si rare et si lente, ce n'est pas le manque d'innovateurs; c'est bien plutôt ce concours de

* Jean-Marie Lucas-Girardville est cocrdannateur du développement rágional au ministere du Tourisme du Québec. circonstances, cette synchronisation de l'idee, du besoin et de la rencontre des intervenants nécessaires.

\section{Qu'en est-il du tourisme?}

Voilà une industrie qui fait appel à tant d'activités et de secteurs différents qu'elle est en mesure de benéficier des innovations de chacun d'eux. C'est aussi une industrie quí évolue en même temps que la sociêtée qui la fait vivre.

L'innovation dans le tourisme se fait par conséquent au rythme de la technologie qui la sert et de la clientèle qu'elle dessert. Pour survivre, l'industrie doit être de plus en plus concurrentielle face aux destinations étrangères. Et le touriste, peut-être plus que d'autres consommateurs, est sensible aux modes et au progres, soit qu'il les épouse, soit qu'il cherche au contraire à les éviter par désir de compensation.

Innover en tourisme, ce sera donc appréhender ces désirs et ces aspirations latentes du voyageur potentiel et tenter de les lui offrir concrètement.

Et c'est lă que surgit la difficulté : comme I'innovation s'exerce tout d'abord dans la marginalité, elle ne peut $s^{\prime}$ offrir le luxe d'un marketing agressif et efficace, se contentant d'un bouche a oreille plus lent.

Si l'innovation est la clé de lá compétitivité, elle ne peut toutefois s'autofinancer qu'à très long terme. C'est pourquoi elle est l'un des domaines du tourisme qui doit être l'objet d'une attention particulière des pouvoirs publics. II y a, dans le tourisme comme dans le secteur manufacturier, des produits et des techniques de pointe dont il faut aider techniquement et financièrement l'émergence par des interventions incitatives auprès des entrepreneurs.

Sur le plan technique, il s'agira d'innovations en matière de transport (nouveaux carburants, nouveaux véhiculesi, de récréation (planches à voile, radeaux pour le rafting, canons à neige), d'hébergement (bed and breakfast, chalets préfabriqués, chaines d'hôtels à prix madiquel. En matiere de services, il pourra s'agir des clientèles nouvelles avec des besoins spécifiques lenfants, personnes âgées, handicapés, femmes voyageant seules etc.).

On s'interroge souvent sur la question de savoir sí l'offre répond à la demande ou si elle la crée : et bien, en tourisme, elle la crèe bien souvent. 